\title{
ON INJECTIVE SHEAVES
}

H. Kleisli and Y.C. Wu

(received June 1, 1963)

Introduction. A divisible abelian group $D$ can be characterized by the following property: Every homomorphism from an abelian group $A$ to $D$ can be extended to every abelian group $B$ containing $A$. This together with the result that every abelian group can be embedded in a divisible group is a crucial point in many investigations on abelian groups. It was Baer, [1], who extended this result to modules over an arbitrary ring, replacing divisible groups by injective modules, that is, modules with the property mentioned above. Another proof was found later by Eckmann and Schopf, [3]. This proof assumes the proposition to hold for abelian groups and transfers it in a very simple and elegant manner to modules. In the sequel, we shall refer to this proof as to the Eckmann-Schopf proof.

One may ask whether the above result is valid in other categories than the category of modules ower a ring, since the concept of an injective module obviously can be formulated categorically, that means, one can define injective objects in an arbitrary category (cf. [2], appendix). It should be mentioned that this question is of considerable importance from the viewpoint of certain constructions in homological algebra. Besides, a category where the above result holds is called a category with enough injectives. Of particular interest are the categories of sheaves on a topological space. A proof that there are enough injectives in the se categories was given by Godement, [4]; another one is due to Grothendieck, [6].

The purpose of this note is to discuss how the EckmannSchopf proof for the existence of enough injective modules can be formulated in such a way that it allows an extension to sheaves, more explicitly, provides a new proof for the existence of enough

Canad. Math. Bull. vol. 7, no. 3, July 1964. 
injective modules over a sheaf of rings on a topological space. In order to do that we shall use the notion of an injective structure and a general transfer theorem for such structures, both due to Maranda, [9]. In the first section, we recall the definition of an injective structure and formulate a special case (suitable for our purpose) of Maranda's transfer theorem. In section 2, we discuss the Eckmann-Schopf proof from the view-point of the transfer of injective structures, and apply it to the categories of abelian sheaves and of modules over a sheaf of rings.

1. Preliminaries. For the definition of the notion category, functor, functor equivalence, etc., as well as for the notation used here, see [6]. A functor $F$ defined on a category $C$ will often be denoted by $F C$. Similarly, if $F$ and $G$ are functors defined on $C$, then a functor morphism (natural transformation) $s$ from $F$ to $G$ will be denoted by $s e: F e \rightarrow G e$.

Let $\mathcal{C}$ be a category, and let $\mathcal{P}$ be a class of its morphisms. An object $C$ of $C$ is called $P$-injective if, for every morphism $u: A \rightarrow B$ which belongs to $\bar{P}$, the induced mapping

$$
u *: \operatorname{Hom}(B, C) \rightarrow \operatorname{Hom}(A, C)
$$

is surjective (onto). The class of all $P$-injectives is denoted by $\phi \mathcal{P}$.

Let $y$ be a class of objects of $C$. A morphism u: $A \rightarrow B$ is called $Y$-proper if, for every object $C$ which belongs to $y$, the induced mapping (1.1) is surjective. The class of all $\boldsymbol{y}$-proper morphisms is denoted by $\psi \boldsymbol{y}$.

Definition (Maranda [9]). A couple $(\mathcal{P}, \boldsymbol{Y}$ ) consisting of a class $P$ of morphisms and of a class $y$ of objects of $C$ is called an injective structure in $C$ if (i) $\mathcal{Y}=\phi \mathcal{P}$, (ii) $P=\psi y$, (iii) for every object $A$ of $C$, there exists a u: $A \rightarrow Q \in \mathcal{P}$ where $Q \in \mathcal{Y}$.

We give only two obvious examples. For more examples and for elementary properties of injective structures see [9].

(a) In every category, the class $P_{0}$ of all retractions and the class $y_{0}$ of all objects form an injective structure 
(in the terminology of [9], the finest injective structure).

(b) If $e$ is an exact (abelian) category with enough injectives, the class $\mathcal{P}_{e}$ of all monomorphisms and the class $Y_{e}$ of all injectives define an injective structure $\left(P_{e}, Y_{e}\right)$, called the exact structure in $C$.

An object $A$ is a retract of an object $B$ if there exist morphisms $u: A \rightarrow B$ and $v: B \rightarrow A$ such that $v u={ }_{A}$. If $Y$ is a class of objects, the class of all retracts of objects in $Y$ is denoted by $P Y$. If the couple $(P, P Y)$ is an injective structure of $\mathcal{C}$, in [9], the class $\boldsymbol{y}$ is called a base of the injectives of $(P, P y)$.

Let $C$ and $e^{\prime}$ be two categories, $T$ a covariant functor from $C$ to $e^{\prime}$ and $S$ a covariant functor in the opposite direction, from $e^{\prime}$ to $e$. Furthermore, let $(P, P Y)$ be an injective structure in $\mathcal{C}$. By means of $S$ and $T$, we construct a couple $\left(\rho^{\prime}, Y^{\prime}\right)$ as follows:

$\rho^{\prime}=S^{-1} \rho$ is the class of morphisms $u^{\prime}: A^{\prime} \rightarrow B^{\prime}$ of $e^{\prime}$ such that $S u^{\prime}: S A^{\prime} \rightarrow S B^{\prime}$ belongs to $P ; y^{\prime}=T y$ is the class of all objects $C^{\prime}$ of the form $C^{\prime}=T C$ for some $C$ which belongs to $\boldsymbol{y}$. (It should be noted that we may restrict ourselves to object functions T.) We ask the question: What conditions are required for $S$ and $T$ such that $\left(P^{\prime}, P y^{\prime}\right)$ is an injective structure in $e^{\prime}$ ? The subsequent theorem contains a sufficient condition: $S$ is the left adjoint of $T$ in the sense of $\mathrm{Kan}$, [8], or equivalently, there exists a functor morphism $\mathrm{k}$ from the identity functor $I$ of $e$ to the composite functor TS such that $(S, k)$ is a regular left adjoint system in the sense of Maranda, [9]. The couple $\left(P^{\prime}, P \mathcal{Y}^{\prime}\right)$ is then called the transferred injective structure in $e^{\prime}$.

THEOREM (transfer theorem). Let $S$ be a covariant functor from $e^{\prime}$ to $e, T$ an object function from $e$ to $e^{\prime}$, and $(P, P Y)$ an injective structure in $C$. If, for every $Q$ of $\mathcal{Y}$, there exists a functor equivalence

$$
\text { s } e^{\prime}: \operatorname{Hom}\left(S e^{\prime}, Q\right) \rightarrow \operatorname{Hom}\left(e^{\prime}, T Q\right) \text {, }
$$


then the couple $\left(P^{\prime}, P Y^{\prime}\right)$, where $P^{\prime}=S^{-1} \mathcal{P}$ and $y^{\prime}=\mathrm{T} y$, is an injective structure in $e^{\prime}$.

This Theorem is due to Maranda and is contained in [9]. Although it is not explicitly stated as a proposition, it follows from the arguments on page 108. Besides, a direct proof can be found without much difficulty.

The following example of functor equivalences leads to a useful corollary of the transfer theorem. Let $e$ be a category with direct products for families of objects indexed by some index set I. As usual, we denote the category of families $A=\left(A_{i}\right)_{i \in I}$ of objects $A_{i}$ of $e$ by $e^{I}$. The function $\Pi$, which assigns to each $A$ of $e^{I}$ the direct product II $_{i \in I}$, has, by definition even (cf. [6], p. 123), the property: For every object $Q$ of $e^{I}$, there exists a functor equivalence

$$
\text { s } e: \operatorname{Hom}(E e, Q) \rightarrow \operatorname{Hom}(C, \Pi Q)
$$

where $E$ is the embedding functor from $e$ to $e^{I}$ which assigns, to every object $C$ of $C$, the family $E C=\left(C_{i}\right)_{i \in I}$ where $C_{i}=C$ for every $i \in I$, and to every morphism $\mathrm{u}: \mathrm{B} \rightarrow \mathrm{C}$ of $\mathcal{C}$, the "mapping" Eu: EB $\rightarrow \mathrm{EC}$ whose "components" $u_{i}=u$ for every $i \in I$.

COROLLARY. Let $\left(P_{i}, P y_{i}\right)_{i \in I}$ be a family of injective structures in $e$. If $e$ possesses direct products for all families $\left(A_{i}\right)_{i \in I}$ of objects $A_{i}$ of $e$, then $P^{\prime}=\bigcap_{i \in I} P_{i}$ and the class $y^{\prime}$ of all direct products $\prod_{i \in I} Q_{i}$, where $Q_{i} \in Y_{i}$ for every $i \in I$, define another injective structure $\left(p^{i}, P y^{\prime}\right)$ in $C$.

This follows immediately from the transfer theorem and the observation that the couple $(P, P y)=\left(\left(P_{i}\right)_{i \in I}, P\left(y_{i}\right)_{i \in I}\right)$ is an injective structure in $e^{I}$. 
2. The Eckmann-Schopf proof. Let $R$ be a ring with identity. Denote by $\mathcal{H}$ the category of unitary right modules over R, briefly, $R$-modules, and by $A$ the category of abelian groups or $Z$-modules ( $Z$ denotes the ring of integers). The well-known as sociativity formula (cf. [2], p. 28)

$$
\operatorname{Hom}_{Z}\left(A \otimes_{R} B, C\right) \approx \operatorname{Hom}_{R}\left(A, \operatorname{Hom}_{Z}(B, C)\right)
$$

implies that, for every $Q$ of $A$, there exists a functor equivalence

$$
\text { s } \mathcal{H} \operatorname{Hom}_{z}\left(\mathcal{H} \otimes_{R} R, Q\right) \rightarrow \operatorname{Hom}_{R}\left(\mathcal{H}, \operatorname{Hom}_{Z}(R, Q)\right.
$$

where the ring $R$ is considered as left $R$-module. The set $T Q=\operatorname{Hom}_{Z}(R, Q)$ with the usual (right) R-module structure is known as the contravariant $\varphi$-extension of $Q$ ( $\varphi$ being the natural embedding of $Z$ in $R$ ). The covariant functor $s \mu=\mu \otimes_{R} R$ is the functor which assigns to each $R$-module its additive group, and to each R-homomorphism the corresponding group homomorphism.

Let $\left(Y_{e}, P_{e}\right)$ be the exact structure in $\mu$ (cf. example (b), section 1). It should be noted that we assume here that $A$ has enough injectives. By the transfer theorem, the functor equivalences (2.2) imply that the couple $\left(P^{\prime}, P y^{\prime}\right)$ given by $P_{1}=S^{-1} \mathcal{P}_{e}$ and $y_{1}=T y_{e}$ is an injective structure in $\mathcal{H}$. Clearly, an R-homomorphism $u: A \rightarrow B$ is an $R$-monomorphism if and only if it is a monomorphism of the additive groups of $A$ and $B$. In other words, $u$ is a monomorphism of $\mu$ if and only if Su belongs to $\mathcal{P}_{e}$. Thus, $P_{1}$ is the class of all $\mathrm{R}$-monomorphisms. This, together with the result that $\left(\mathcal{P}^{\prime}, P y^{\prime}\right)$ is an injective structure in $\mu$, implies that the category $\mathcal{H}$ has enough injectives.

The above proof for the existence of enough injective $R$-modules is just a reformulation of the Eckmann-Schopf proof (cf. [3]; see also [9], p. 108). However, this reformulation is done in such a way that it can be applied immediately to more general situations such as the one where $\mathcal{H}$ is replaced by the 
category of abelian sheaves on a topological space.

For the definition of the notion of a sheaf, presheaf, sheaf homomorphism, etc., see [4], chapter II. An abelian (pre)sheaf is a (pre)sheaf with values in the category $A$ of abelian groups ( $Z$-modules). The category of abelian sheaves on a topological space $X$ is denoted by $\rho(x, z)$. It is not difficult to see that $\rho(X, Z)$ is a category with arbitrary direct products.

For each point $x \in X$, define an object function $E_{x}$ from $A$ to $\rho(x, z)$ as follows: For every $A$ of $A$, put $E_{x} A U=\left\{\begin{array}{ll}A & \text { if } x \in U \\ 0 & \text { otherwise }\end{array} \quad e_{x}^{A} U= \begin{cases}{ }^{1} A & \text { if } x \in U \\ 0 & \text { otherwise }\end{cases}\right.$ where $U$ and $V$ are open sets of $X$ subject to the condition $U C V . \quad$ Clearly, the system $E_{x} A=\left\{E_{x} A U, e_{x} A_{U}^{V}\right\}$ defines an abelian presheaf on $X$; it is easy to verify that it is even a sheaf. Furthermore, let $S_{x}$ be the covariant functor from $\rho(x, z)$ to $A$. which assigns to each sheaf $F=\left\{F U, f_{U}^{V}\right\}$ $\left(f_{U}^{V}\right.$ denotes the restriction from $F V$ to $\left.F U\right)$ its stalk $S_{x} F$ at $x$, that is, the abelian group

$$
\text { S }_{x} F=\underset{U \geqslant x}{\operatorname{dir} .} \lim .\left\{F U, f_{U}^{V}\right\}
$$

and to each sheaf homomorphism in an obvious way a group homomorphism. It follows from the definition of a direct limit (cf. [8], p. 309) that, for every abelian group $Q$, there exists a functor equivalence

(2. 3) $s_{x} \rho(x, Z): \operatorname{Hom}\left(S_{x} \rho(X, Z), Q\right) \rightarrow \operatorname{Hom}\left(\rho(X, Z), E_{x} Q\right) \cdot$

PROPOSITION 1. The category $\rho(x, z)$ of abelian sheaves on $X$ has enough injectives.

Proof. (Compare with the above arguments in the 
category $\mathcal{Y})$. Let $\left(Y_{e}, \rho_{e}\right)$ be the exact structure in $\mathcal{A}$. By the transfer theorem, the functor equivalences (2. 3) imply that, for every $x \in X$, the couple $\left(\rho_{x}, P y_{x}\right)$ given by $P_{x}=s_{x}^{-1} P_{e}$ and $y_{x}=E_{x} y_{e}$ is an injective structure in $\rho(x, z)$. Since $\rho(x, z)$ is a category with arbitrary direct products, by the corollary of the transfer theorem, the couple $\left(P^{\prime}, P^{\prime}\right)$ given by $P_{1}=\cap \rho_{x}$ and the class $Y^{\prime}$ of all $x \in \mathrm{X}$ direct products $\Pi_{x \in X} Q_{x}$ where $Q_{x} \in Y_{x}$ defines another

injective structure in $\rho(x, z)$. A sheaf homomorphism $u: F \rightarrow G$ is a monomorphism of $\rho(X, Z)$ if and only if the homorphisms $S_{x} u: S_{x} F \rightarrow S_{x} G$ are monomorphisms of $A$ for every $x \in X$. In other words, $u$ is a monomorphism of $\rho(x, z)$ if and only if $s_{x} u \in P_{e}$ for every $x \in X$. Thus, $P_{1}$ is the class of all monomorphisms of $\rho(x, z)$. This, together with the result that $\left(P^{\prime}, P Y^{\prime}\right)$ is an injective structure in $\rho(x, z)$, implies that the category $\rho(x, z)$ has enough injectives.

Let $R$ be a sheaf of rings on a topological space $X$. Denote by $\rho(X, R)$ the category of all $R$-modules on $X$, that is, of all abelian sheaves $F$ on $X$ where, for every open set $U$ of $X$, the groups $F U$ have a natural $R U$-module structure (for details, see [4], p. 127). Clearly, for the simple sheaf $Z$ given by the ring of integers, $\rho(x, z)$ is the category of abelian sheaves on $X$.

The as sociativity formula (2.1) for modules over an ordinary ring is easily seen to be valid when $R$ is a sheaf of rings, $Z$ the simple sheaf given by the ring of integers, and $A, B$ and $C$ appropriate modules over $R$ and $Z$ (with fixed base space $X)$. Hence, for every abelian sheaf $Q$ on $X$, there exists a functor equivalence

(2. 4) s $\rho(X, R): \operatorname{Hom}\left(\rho(X, R) \otimes_{R} R, Q\right) \rightarrow \operatorname{Hom}(\rho(X, R), \operatorname{Hom}(R, Q$

where the sheaf $R$ is considered as left $R$-module over $X$, and Hom $(R, Q)$ is in the usual way considered as an $R$-module on $X$ (cf. [4], p. 128). The covariant functor $S \rho(x, R)=\rho(x, R) \otimes_{R} R$ 
is the functor which assigns to each $R$-module on $X$ its underlying abelian sheaf, and to each $R$-homomorphism the corresponding sheaf homomorphism.

PROPOSITION 2. The category $\rho(X, R)$ of $R$-modules on $\mathrm{X}$ has enough injectives.

Proof. Let $\left(P^{\prime}, P y^{\prime}\right)$ be the exact structure in $\rho(x, Z)$ (which exists by proposition 1). By the transfer theorem, the natural equivalences (2.4) imply that the couple $(\rho ", P y ")$ given by $\rho "=S^{-1} \rho$, and $y "=\operatorname{Hom}\left(R, y^{\prime}\right)$ is an injective structure in $\rho(X, R)$. By the same arguments as in the case of modules over an ordinary ring, $\rho$ " is seen to be the class of all monomorphisms of $\rho(X, R)$. From this, the assertion follows.

Finally, a remark concerning the category of sheaves with values in an abelian category. Under additional conditions for the value-category, such as requiring it to be a right perfect category (Grothendieck category) with enough small objects (cf. [5]), or with a Noetherian projective generator (cf. [7]), the Eckmann-Schopf proof, as formulated for proposition 1, works equally well. * The crucial point, where the additional conditions are exploited, consists in proving that $P^{\prime}$ is the class of all monomorphisms. It should be mentioned, however, that even without all these conditions, the morphisms of $\mathcal{P}$, are apt to replace monomorphisms from the viewpoint of most homological constructions.

* These results have been established by $Y_{0}-C$. Wu and are contained in his Master's thesis 'Injective sheaves with values in a category" (University of Ottawa). 


\section{REFERENCES}

1. R. Baer, Abelian groups that are direct summands of every containing abelian group, Bull. Amer. Math. Soc. $46(1940), 800-806$.

2. H. Cartan and S. Eilenberg, Homological algebra, Princeton University Press, 1956.

3. B. Eckmann und A. Schopf, Über injective Moduln, Archiv der Math. 4 (1953), 75-78.

4. R. Godement, Topologie algébrique et théorie des faisceaux, Act. Sci. et Ind. 1252, Hermann Paris, 1958.

5. J. W. Gray, Sheaves with values in a category (Mimeographed, Columbia University).

6. A. Grothendieck, Sur quelques points d'algebre homologique, Tôhoku Math. J. 9 (1957), 119-221.

7. A. Heller and K.A. Rowe, On the category of sheaves, Amer. J. Math. 84 (1962), 205-216.

8. D. M. Kan, Adjoint functors, Trans. Amer. Math. Soc. 87 (1958), 294-329.

9. J. M. Maranda, Injective structures, Trans. Amer. Math. Soc. 110 (1964), 98-135.

University of Ottawa 